\title{
Well-posedness for parametric strong vector quasi-equilibrium problems with applications
}

\author{
Qiu-ying Li ${ }^{1}$ and San-hua Wang ${ }^{2^{*}}$
}

\author{
* Correspondence: wsh_315@163. \\ com \\ 2Department of Mathematics, \\ Nanchang University, Nanchang, \\ Jiangxi 330031, People's Republic \\ of China \\ Full list of author information is \\ available at the end of the article
}

\begin{abstract}
In this article, we generalize the concept of well-posedness to the parametric strong vector quasi-equilibrium problem. Under some suitable conditions, we establish some characterizations of well-posedness for parametric strong vector quasiequilibrium problems. The corresponding concept of well-posedness in the generalized sense is also investigated for the parametric strong vector quasiequilibrium problem. As applications, we investigate the well-posedness for strong vector quasi-variational inequality problems and strong vector quasi-optimization problems.
\end{abstract}

2000 Mathematics subject classification: 49K40; 90C31.

Keywords: parametric strong vector quasi-equilibrium problem, well-posedness, approximating sequence, upper semicontinuity; lower semicontinuity

\section{Introduction}

Equilibrium problem was first introduced by Blum and Oettli [1], which includes optimization problems, fixed point problems, variational inequality problems, and complementarity problems as special cases. During the recent years, equilibrium problem has been extensively studied and generalized (e.g., [2,3]).

It is well known that well-posedness is very important for both optimization theory and numerical methods of optimization problems, which guarantees that, for every approximating solution sequence, there is a subsequence which converges to a solution. Well-posedness of unconstrained and constrained scalar optimization problems was first introduced and studied by Tykhonov [4] and Levitin and Polyak [5], respectively. Since then, various concepts of well-posedness have been introduced and extensively studied for minimization problems and vector optimization problems. For details, we refer readers to [6-13] and the references therein.

In recent years, the concept of well-posedness has been generalized to several related problems: variational inequality problems [14-18], saddle point problems [19], Nash equilibrium problems [16,20-23], inclusion problems [24,25], and fixed point problems [24-26].

Recently Fang et al. [27] generalized the concepts of well-posedness to equilibrium problems and to optimization problems with equilibrium constraints and gave some metric characterizations of well-posedness for equilibrium and optimization problems with equilibrium constraints. Subsequently, the well-posedness has been extensively studied by many authors for various equilibrium problems, such as scalar equilibrium

(C) $2011 \mathrm{Li}$ and Wang; licensee Springer. This is an Open Access article distributed under the terms of the Creative Commons Attribution License (http://creativecommons.org/licenses/by/2.0), which permits unrestricted use, distribution, and reproduction in any medium, provided the original work is properly cited. 
problems [28], vector equilibrium problems [29-31], parametric vector equilibrium problems [32], vector quasi-equilibrium problems [33], generalized vector quasi-equilibrium problems [34], and symmetric quasi-equilibrium problems [35]. It is worth mentioning that the most of results of well-posedness are for scalar equilibrium problem or for weak vector equilibrium problem which depends on int $C \neq \varnothing$. However, in many cases, the cone $C$ has an empty interior. For example, in the classical Banach spaces $l^{p}$ and $L^{p}(\Omega)$, where $1<p<\infty$, the standard ordering cone has an empty interior. In this case, we cannot study the well-posedness for the weak vector equilibrium problem. However, we can study the strong vector equilibrium problem since the strong vector equilibrium problem does not need int $C \neq \varnothing$. On the other hand, to the best of our knowledge, the result of well-posedness for the strong vector equilibrium problem is very few.

On the other hand, the strong vector equilibrium problem is a class of important equilibrium problems since a solution for the strong vector equilibrium problem is an ideal solution, which is better than other solutions such as efficient solution, weak efficient solution, Henig efficient solution and supper efficient solution (e.g., [36]). Thus, it is important to investigate the well-posedness for strong vector equilibrium problems.

Motivated and inspired by the studies mentioned above, in this article, we introduce and study the well-posedness for parametric strong vector quasi-equilibrium problems. Under suitable conditions, we obtain some necessary and sufficient conditions for the well-posedness of parametric strong vector quasi-equilibrium problems. As applications, we investigate the well-posedness for strong vector quasi-variational inequality problems and strong vector quasi-optimization problems.

\section{Preliminaries}

Throughout this article, unless otherwise specified, we use the following notations. Let $X$ be a nonempty closed subset of a metric space $(E, d)$; $Y$ be a Huasdorff topological vector space; and $\Lambda$ and $P$ be two nonempty closed subsets of two metric spaces, respectively. Let $K: \Lambda \times X \rightarrow 2^{X}$ and $C: \Lambda \times X \rightarrow 2^{Y}$ be set-valued mappings such that, for each $(\lambda, x) \in \Lambda \times X, C(\lambda, x)$ is a nonempty closed convex cone in $Y$. Let $e: X$ $\rightarrow Y$ be a continuous mapping such that, for each $x \in X$ and $\lambda \in \Lambda, e(x) \in C(\lambda, x)$. Let $f: P \times X \times X \rightarrow Y$ be a vector-valued mapping. For any fixed $(\lambda, p) \in \Lambda \times P$, the parametric strong vector quasi-equilibrium problem (for short, (PSVQEP) $)_{, p}$ ) is to find $\bar{x} \in X$ such that $\bar{x} \in K(\lambda, \bar{x})$ and

$$
f(p, \bar{x}, y) \in C(\lambda, \bar{x}), \quad \forall y \in K(\lambda, \bar{x}) .
$$

Denote by (PSVQEP) the family $\left\{(\operatorname{PSVQEP})_{\lambda, p}:(\lambda, p) \in \Lambda \times P\right\}$. For each $(\lambda, p) \in \Lambda$ $\times P$, let $S(\lambda, p)$ be the solution set for (PSVQEP) $)_{\lambda, p}$, i.e.,

$$
S(\lambda, p)=\{x \in X: x \in K(\lambda, x) \text { and } f(p, x, y) \in C(\lambda, x), \quad \forall y \in K(\lambda, x)\} .
$$

Remark 2.1. If $E$ is a real Banach space, $Y=R, f: P \times X \times X \rightarrow R, K(\lambda, x)=X$ and $C$ $(\lambda, x)=(0,+\infty)$ for all $(\lambda, x) \in \Lambda \times X$, then (PSVQEP) $)_{\lambda, p}$ reduces to the following parametric equilibrium problem (for short, $(\mathrm{PEP})_{p}$ ): find $\bar{x} \in X$ such that

$$
f(p, \bar{x}, y) \geq 0, \quad \forall y \in X,
$$

which has been studied in [27]. 
Definition 2.1. Let $(\lambda, p) \in \Lambda \times P$ and $\left\{\left(\lambda_{n}, p_{n}\right)\right\} \subseteq \Lambda \times P$ with $\left(\lambda_{n}, p_{n}\right) \rightarrow(\lambda, p)$. A sequence $\left\{x_{n}\right\} \subseteq X$ is said to be approximating for (PSVQEP) $)_{\lambda, p}$ corresponding to $\left\{\left(\lambda_{n}\right.\right.$, $\left.\left.p_{n}\right)\right\}$ if there exists a sequence $\left\{\varepsilon_{n}\right\} \subseteq R^{+}$with $\varepsilon_{n} \rightarrow 0$ such that, for each $n \in N$,

$$
x_{n} \in K\left(\lambda_{n}, x_{n}\right)
$$

and

$$
f\left(p_{n}, x_{n}, y\right)+\varepsilon_{n} e\left(x_{n}\right) \in C\left(\lambda_{n}, x_{n}\right), \quad \forall y \in K\left(\lambda_{n}, x_{n}\right) .
$$

Definition 2.2. (PSVQEP) is said to be well-posed if, for every $(\lambda, p) \in \Lambda \times P$,

(i) (PSVQEP) $)_{\lambda, p}$ has a unique solution $x_{\lambda, p}$, i.e., $S(\lambda, p)=\left\{x_{\lambda, p}\right\}$;

(ii) for any sequence $\left\{\left(\lambda_{n}, p_{n}\right)\right\} \subseteq \Lambda \times P$ with $\left(\lambda_{n}, p_{n}\right) \rightarrow(\lambda, p)$, every approximating sequence $\left\{x_{n}\right\}$ for (PSVQEP) $)_{\lambda, p}$ corresponding to $\left\{\left(\lambda_{n}, p_{n}\right)\right\}$ converges to $x_{\lambda, p}$.

Definition 2.3. (PSVQEP) is said to be well-posed in the generalized sense if, for every $(\lambda, p) \in \Lambda \times P$,

(i) $S(\lambda, p) \neq \varnothing$;

(ii) for any sequence $\left\{\left(\lambda_{n}, p_{n}\right)\right\} \subseteq \Lambda \times P$ with $\left(\lambda_{n}, p_{n}\right) \rightarrow(\lambda, p)$, every approximating sequence $\left\{x_{n}\right\}$ for (PSVQEP $)_{\lambda, p}$ corresponding to $\left\{\left(\lambda_{n}, p_{n}\right)\right\}$ has a subsequence which converges to some point of $S(\lambda, p)$.

Remark 2.2. When (PSVQEP) ${ }_{\lambda, p}$ reduces to (PEP) $)_{p}$, Definition 2.1 coincides with 3.1 of [27], and Definitions 2.2 and 2.3 coincide with 3.2 of [27].

Definition 2.4. Let $\Lambda, P$, and $X$ be topological spaces and $Y$ be a topological vector space. Let $C: \Lambda \times X \rightarrow 2^{Y}$ be a set-valued mapping such that, for each $(\lambda, x) \in \Lambda \times X$, $C(\lambda, x)$ is a nonempty closed convex cone in $Y$. Let $f: P \times X \times X \rightarrow Y$ be a vectorvalued mapping. For any fixed $(\lambda, x) \in \Lambda \times X, f$ is said to be upper (resp. lower) $C(\lambda$, $x$ ) continuous if, for any $(p, y) \in P \times X$ and any neighborhood $V$ of 0 in $Y$, there exist neighborhoods $U_{p}, U_{x}$ and $U_{y}$ of $p, x$, and $y$, respectively, such that

$$
\begin{gathered}
f\left(p^{\prime}, x^{\prime}, y^{\prime}\right) \in f(p, x, y)+V+C(\lambda, x), \quad \forall\left(p^{\prime}, x^{\prime}, y^{\prime}\right) \in U_{p} \times U_{x} \times U_{y} . \\
\text { (resp. } \left.f\left(p^{\prime}, x^{\prime}, y^{\prime}\right) \in f(p, x, y)+V-C(\lambda, x), \quad \forall\left(p^{\prime}, x^{\prime}, y^{\prime}\right) \in U_{p} \times U_{x} \times U_{y} .\right)
\end{gathered}
$$

Remark 2.3. If $f: P \times X \times X \rightarrow Y$ is continuous, then for any fixed $(\lambda, x) \in \Lambda \times X, f$ is both upper $C(\lambda, x)$ continuous and lower $C(\lambda, x)$ continuous.

Definition 2.5 [37]. Let $X$ and $Y$ be two topological spaces. A set-valued mapping $T$ : $X \rightarrow 2^{Y}$ is said to be

(i) upper semicontinuous (for short, u.s.c.) at $x_{0} \in X$ if, for each open set $V$ in $Y$ with $T\left(x_{0}\right) \subseteq V$, there exists an open neighborhood $U\left(x_{0}\right)$ of $x_{0}$ such that $T(x) \subseteq V$ for all $x \in U\left(x_{0}\right)$;

(ii) lower semicontinuous (for short, l.s.c.) at $x_{0} \in X$ if, for each open set $V$ in $Y$ with $T\left(x_{0}\right) \cap V \neq \varnothing$, there exists an open neighborhood $U\left(x_{0}\right)$ of $x_{0}$ such that $T(x)$ $\cap V \neq \varnothing$ for all $x \in U\left(x_{0}\right)$;

(iii) u.s.c. (resp. l.s.c.) on $X$ if it is u.s.c. (resp. l.s.c.) at every point $x \in X$; 
(iv) continuous on $X$ if it is both u.s.c. and l.s.c. on $X$;

(v) closed if the graph $\operatorname{Gr}(T)=\{(x, y) \in X \times Y: y \in T(x)\}$ is a closed subset of $X \times Y$.

Lemma 2.1 [37]. Let $X$ and $Y$ be two topological spaces, and $F: X \rightarrow 2^{Y}$ a set-valued mapping.

(i) If $F$ is u.s.c. and close-valued, then $F$ is closed;

(ii) If $F(x)$ is a compact set, then $F$ is u.s.c. at $x \in X$, if and only if for any net $\left\{x_{\alpha}\right\}$ $\subseteq X$ with $x_{\alpha} \rightarrow x$ and any net $\left\{y_{\alpha}\right\} \subseteq Y$ with $y_{\alpha} \in F\left(x_{\alpha}\right)$ for all $\alpha$, there exist $y \in F$ $(x)$ and a subnet $\left\{y_{\beta}\right\}$ of $\left\{y_{\alpha}\right\}$ such that $y_{\beta} \rightarrow y$;

(iii) $F$ is l.s.c. at $x \in X$, if and only if for any $y \in F(x)$ and for any net $\left\{x_{\alpha}\right\}$ with $x_{\alpha}$ $\rightarrow x$, there exists a net $\left\{y_{\alpha}\right\}$ with $y_{\alpha} \in F\left(x_{\alpha}\right)$ for all $\alpha$ such that $y_{\alpha} \rightarrow y$.

We also need the concepts of noncompactness measure and Hausdorff metric.

Definition 2.6 [38]. Let $E$ be completed. The Kuratowski measure of noncompactness of a set $A \subseteq E$ is defined by

$$
\mu(A)=\inf \left\{\varepsilon>0: A \subset \cup_{i=1}^{n} A_{i}, \quad \operatorname{diam} A_{i}<\varepsilon, i=1,2, \ldots, n\right\},
$$

where $\operatorname{diam} A$ denotes the diameter of $A$ defined by $\operatorname{diam} A=\sup \left\{d\left(x_{1}, x_{2}\right): x_{1}, x_{2} \in\right.$ A\}.

Definition 2.7 [38]. Let $A$ and $B$ be nonempty subsets of $E$. The Hausdorff metric $H$ $(, \cdot)$ between $A$ and $B$ is defined by

$$
H(A, B)=\max \{h(A, B), h(B, A)\},
$$

where $h(A, B)=\sup _{a \in A} d(a, B)$ with $d(a, B)=\inf _{b \in B} d(a, b)$.

\section{Well-posedness for (PSVQEP)}

In this section, we shall establish some characterizations of well-posedness for (PSVQEP).

\subsection{Continuity characterization of well-posedness for (PSVQEP)}

For each $(\lambda, p) \in \Lambda \times P$ and $\varepsilon \geq 0$, the $\varepsilon$-solution set for (PSVQEP) $)_{\lambda, p}$ is defined by

$$
\Pi(\lambda, p, \varepsilon):=\{x \in X: x \in K(\lambda, x) \text { and } f(p, x, y)+\varepsilon e(x) \in C(\lambda, x), \quad \forall y \in K(\lambda, x)\} .
$$

We call $\Pi$ an $\varepsilon$-solution mapping.

Clearly, for each $(\lambda, p) \in \Lambda \times P$ and $\varepsilon \geq 0, S(\lambda, p) \subseteq \Pi(\lambda, p, \varepsilon)$, and $S(\lambda, p)=\Pi(\lambda, p$, $0)$.

Lemma 3.1. For any fixed $(\lambda, x) \in \Lambda \times X$, if $f$ is lower $C(\lambda, x)$ continuous, then for every $\varepsilon \geq 0, f(p, x, y)+\varepsilon e(x)$ is lower $C(\lambda, x)$ continuous.

Proof. Let $(\lambda, x) \in \Lambda \times X$ be such that $f$ is lower $C(\lambda, x)$ continuous. For any given $\varepsilon$ $\geq 0$, define a vector-valued mapping $F: P \times X \times X \rightarrow Y$ as follows:

$$
F(p, x, y)=f(p, x, y)+\varepsilon e(x), \quad \forall(p, x, y) \in P \times X \times X .
$$

For any neighborhood $V$ of 0 in $Y$, there exists a balanced neighborhood $V_{1}$ of 0 in $Y$ such that 


$$
V_{1}+V_{1} \subseteq V
$$

For any $(p, y) \in P \times X$, since $e$ is continuous and $f$ is lower $C(\lambda, x)$ continuous, there exist neighborhoods $U_{p}, U_{x}$ and $U_{y}$ of $p, x$, and $y$, respectively, such that

$$
f\left(p^{\prime}, x^{\prime}, y^{\prime}\right) \in f(p, x, y)+V_{1}-C(\lambda, x), \quad \forall\left(p^{\prime}, x^{\prime}, y^{\prime}\right) \in U_{p} \times U_{x} \times U_{y} .
$$

and

$$
\varepsilon e\left(x^{\prime}\right) \in \varepsilon e(x)+V_{1}, \quad \forall x^{\prime} \in U_{x} .
$$

Let $U=U_{p} \times U_{x} \times U_{y}$, then by (3.2), (3.3), and (3.1), we have, for each $\left(p^{\prime}, x^{\prime}, y^{\prime}\right) \in$ $U$,

$$
\begin{aligned}
F\left(p^{\prime}, x^{\prime}, y^{\prime}\right) & =f\left(p^{\prime}, x^{\prime}, y^{\prime}\right)+\varepsilon e\left(x^{\prime}\right) \\
& \in f(p, x, y)+V_{1}-C(\lambda, x)+\varepsilon e(x)+V_{1} \\
& \subseteq f(p, x, y)+\varepsilon e(x)+V-C(\lambda, x) \\
& =F(p, x, y)+V-C(\lambda, x) .
\end{aligned}
$$

that is,

$$
F\left(p^{\prime}, x^{\prime}, y^{\prime}\right) \in F(p, x, y)+V-C(\lambda, x), \quad \forall\left(p^{\prime}, x^{\prime}, y^{\prime}\right) \in U .
$$

Thus, $F$ is lower $C(\lambda, x)$ continuous. This completes the proof.

Lemma 3.2. Assume that

(i) for each $\lambda \in \Lambda, K(\lambda, \cdot)$ is continuous and close-valued on $X$;

(ii) for each $\lambda, C(\lambda, \cdot)$ is u.s.c. on $X$;

(iii) for each $(\lambda, x) \in \Lambda \times X, f$ is lower $C(\lambda, x)$ continuous.

Then for each $(\lambda, p, \varepsilon) \in \Lambda \times P \times R^{+}, S(\lambda, p)$ and $\Pi(\lambda, p, \varepsilon)$ are closed subsets of $X$.

Proof. (1) Let $(\lambda, p) \in \Lambda \times P$ be any given sequence. Let $\left\{x_{n}\right\} \subseteq S(\lambda, p)$ be any sequence such that $x_{n} \rightarrow x \in X$. Then, for each $n$, we have

$$
x_{n} \in K\left(\lambda, x_{n}\right)
$$

and

$$
f\left(p, x_{n}, y\right) \in C\left(\lambda, x_{n}\right), \quad \forall y \in K\left(\lambda, x_{n}\right) .
$$

Since $K(\lambda, \cdot)$ is u.s.c. and close-valued, $K(\lambda, \cdot)$ is closed and so $x \in K(\lambda, x)$. For each $y$ $\in K(\lambda, x)$, since $K(\lambda, \cdot)$ is l.s.c. at $x$, there exists a sequence $\left\{y_{n}\right\}$ with $y_{n} \in K\left(\lambda, x_{n}\right)$ such that $y_{n} \rightarrow y$. Then, by (3.4), we have

$$
f\left(p, x_{n}, y_{n}\right) \in C\left(\lambda, x_{n}\right), \quad \forall n \in N .
$$

For any neighborhood $V$ of 0 in $Y$, there exists a balanced neighborhood $V_{0}$ of 0 in $Y$ such that

$$
V_{0}+V_{0} \subseteq V .
$$

For $V_{0}$, since $C(\lambda, \cdot)$ is u.s.c. at $x$ and $f$ is lower $C(\lambda, x)$ continuous, there exist neighborhoods $U_{x}$ and $U_{y}$ of $x$ and $y$, respectively, such that 


$$
\begin{aligned}
& C\left(\lambda, x^{\prime}\right) \subseteq C(\lambda, x)+V_{0}, \quad \forall x^{\prime} \in U_{x} . \\
& f\left(p, x^{\prime}, y^{\prime}\right) \in f(p, x, y)+V_{0}-C(\lambda, x), \quad \forall\left(x^{\prime}, y^{\prime}\right) \in U_{x} \times U_{y} .
\end{aligned}
$$

Since $x_{n} \rightarrow x$ and $y_{n} \rightarrow y$, there exists $n_{0} \in N$ such that, for all $n \geq n_{0}, x_{n} \in U_{x}$, and $y_{n} \in U_{y}$. Then, by (3.7) and (3.8), for all $n \geq n_{0}$,

$$
\begin{aligned}
& C\left(\lambda, x_{n}\right) \subseteq C(\lambda, x)+V_{0} \\
& f\left(p, x_{n}, y_{n}\right) \in f(p, x, y)+V_{0}-C(\lambda, x) .
\end{aligned}
$$

Noting that $V_{0}$ is balanced, we have $-V_{0}=V_{0}$. Then, by (3.10), (3.5), (3.9), and (3.6), we have, for each $n \geq n_{0}$,

$$
\begin{aligned}
f(p, x, y) & \in f\left(p, x_{n}, y_{n}\right)-V_{0}+C(\lambda, x) \\
& =f\left(p, x_{n}, y_{n}\right)+V_{0}+C(\lambda, x) \\
& \subseteq C\left(\lambda, x_{n}\right)+V_{0}+C(\lambda, x) \\
& \subseteq C(\lambda, x)+V_{0}+V_{0}+C(\lambda, x) \\
& \subseteq C(\lambda, x)+V .
\end{aligned}
$$

By the arbitrary of $V$, we get

$$
f(p, x, y) \in C(\lambda, x) \text {. }
$$

Thus, $x \in S(\lambda, p)$, and this implies that $S(\lambda, p)$ is closed.

(2) For each $\varepsilon>0$, let

$$
F(p, x, y)=f(p, x, y)+\varepsilon e(x), \quad \forall(p, x, y) \in P \times X \times X .
$$

Then, by Lemma 3.1, for each $(\lambda, x) \in \Lambda \times X, F$ is lower $C(\lambda, x)$ continuous. It follows from (1) that, for each $(\lambda, p) \in \lambda \times P$, the set

$$
\{x \in X: x \in K(\lambda, x) \text { and } F(p, x, y) \in C(\lambda, x), \forall y \in K(\lambda, x)\}
$$

is closed, i.e., $\Pi(\lambda, p, \varepsilon)$ is closed. This completes the proof.

The following theorem gives a characterization of the well-posedness in the generalized sense for (PSVQEP).

Theorem 3.1. (PSVQEP) is well-posed in the generalized sense, if and only if for every $(\lambda, p) \in \Lambda \times P, S(\lambda, p)$ is a nonempty compact subset of $X$ and $\Pi$ is u.s.c. at $(\lambda, p, 0)$.

Proof. For any $(\lambda, p) \in \Lambda \times P$, suppose that $S(\lambda, p)$ is a nonempty compact subset of $X$ and $\Pi$ is u.s.c. at $(\lambda, p, 0)$. Let $\left\{\left(\lambda_{n}, p_{n}\right)\right\} \subseteq \Lambda \times P$ be an arbitrary sequence with $\left(\lambda_{n}\right.$, $\left.p_{n}\right) \rightarrow(\lambda, p)$ and $\left\{x_{n}\right\}$ be an approximating sequence of (PSVQEP) $)_{\lambda, p}$ corresponding to $\left\{\left(\lambda_{n}, p_{n}\right)\right\}$. Then, there exists a sequence $\left\{\varepsilon_{n}\right\} \subseteq R^{+}$with $\varepsilon_{n} \rightarrow 0$ such that, for each $n \in$ $N$,

$$
x_{n} \in K\left(\lambda_{n}, x_{n}\right)
$$

and

$$
f\left(p_{n}, x_{n}, y\right)+\varepsilon_{n} e\left(x_{n}\right) \in C\left(\lambda_{n}, x_{n}\right), \quad \forall y \in K\left(\lambda_{n}, x_{n}\right) .
$$


Thus, $x_{n} \in \Pi\left(\lambda_{n}, p_{n}, \varepsilon_{n}\right)$. Note that $\Pi(\lambda, p, 0)=S(\lambda, p)$ is compact. Since $\Pi$ is u.s.c. at $(\lambda, p, 0)$, there exists a subsequence $\left\{x_{n_{j}}\right\}$ of $\left\{x_{n}\right\}$ which converges to some point $x_{0} \in$ $\Pi(\lambda, p, 0)=S(\lambda, p)$. Therefore, (PSVQEP) is well-posed in the generalized sense.

Conversely, suppose that (PSVQEP) is well-posed in the generalized sense. Then, for each $(\lambda, p) \in \Lambda \times P, S(\lambda, p)$ is nonempty and compact, and so $\Pi(\lambda, p, 0)$ is nonempty and compact. Let $\left\{\left(\lambda_{n}, p_{n}, \varepsilon_{n}\right)\right\} \subseteq \Lambda \times P \times R^{+}$be an arbitrary sequence with $\left(\lambda_{n}, p_{n}, \varepsilon_{n}\right)$ $\rightarrow(\lambda, p, 0)$ and $x_{n} \in \Pi\left(\lambda_{n}, p_{n}, \varepsilon_{n}\right)$. Then, for each $n \in N$,

$$
x_{n} \in K\left(\lambda_{n}, x_{n}\right)
$$

and

$$
f\left(p_{n}, x_{n}, y\right)+\varepsilon_{n} e\left(x_{n}\right) \in C\left(\lambda_{n}, x_{n}\right), \quad \forall y \in K\left(\lambda_{n}, x_{n}\right) .
$$

Thus, $\left\{x_{n}\right\}$ is an approximating sequence of (PSQVEP) $)_{\lambda, p}$ corresponding to $\left\{\left(\lambda_{n}, p_{n}\right)\right\}$. By the well-posedness in the generalized sense of (PSVQEP), $\left\{x_{n}\right\}$ has a subsequence $\left\{x_{n_{j}}\right\}$ which converges to some point of $S(\lambda, p)=\Pi(\lambda, p, 0)$. Thus, $\Pi$ is u.s.c. at $(\lambda, p$, $0)$. This completes the proof.

For the well-posedness of (PSVQEP), we have the following result.

Theorem 3.2. (PSVQEP) is well-posed, if and only if for every $(\lambda, p) \in \Lambda \times P, S(\lambda, p)$ has a unique point and $\Pi$ is u.s.c. at $(\lambda, p, 0)$.

Proof. The necessity is a direct conclusion of Definition 2.2 and Theorem 3.1.

For the sufficiency, suppose that for any $(\lambda, p) \in \Lambda \times P, S(\lambda, p)$ has a unique point $x_{\lambda, p}$ and $\Pi$ is u.s.c. at $(\lambda, p, 0)$. Let $\left\{\left(\lambda_{n}, p_{n}\right)\right\} \subseteq \Lambda \times P$ be an arbitrary sequence with $\left(\lambda_{n}, p_{n}\right) \rightarrow(\lambda, p)$ and $\left\{x_{n}\right\}$ be an approximating sequence of (PSVQEP) $)_{\lambda, p}$ corresponding to $\left\{\left(\lambda_{n}, p_{n}\right)\right\}$. Then, there exists a sequence $\left\{\varepsilon_{n}\right\} \subseteq R^{+}$with $\varepsilon_{n} \rightarrow 0$ such that, for each $n$ $\in N$,

$$
x_{n} \in K\left(\lambda_{n}, x_{n}\right)
$$

and

$$
f\left(p_{n}, x_{n}, \gamma\right)+\varepsilon_{n} e\left(x_{n}\right) \in C\left(\lambda_{n}, x_{n}\right), \quad \forall y \in K\left(\lambda_{n}, x_{n}\right) .
$$

Thus, $x_{n} \in \Pi\left(\lambda_{n}, p_{n}, \varepsilon_{n}\right)$. Note that $\Pi(\lambda, p, 0)=S(\lambda, p)=\left\{x_{\lambda, p}\right\}$ is a singleton set and so is compact. Since $\Pi$ is u.s.c. at $(\lambda, p, 0)$, for any neighborhood $V$ of $x_{\lambda, p}$, there exists a neighborhood $U$ of $(\lambda, p, 0)$ such that

$$
\Pi\left(\lambda^{\prime}, p^{\prime}, \varepsilon^{\prime}\right) \subseteq V, \quad \forall\left(\lambda^{\prime}, p^{\prime}, \varepsilon^{\prime}\right) \in U .
$$

Since $\left(\lambda_{n}, p_{n}, \varepsilon_{n}\right) \rightarrow(\lambda, p, 0)$, there exists $n_{0} \in N$ such that

$$
\left(\lambda_{n}, p_{n}, \varepsilon_{n}\right) \in U, \quad \forall n \geq n_{0} .
$$

It follows that

$$
x_{n} \in \Pi\left(\lambda_{n}, p_{n}, \varepsilon_{n}\right) \subseteq V, \quad \forall n \geq n_{0} .
$$

Hence $x_{n} \rightarrow x_{\lambda, p}$, and this implies that (PSVQEP) is well-posed. This completes the proof.

Now we give a sufficient condition for the upper semicontinuity of the $\varepsilon$-solution mapping. 
Theorem 3.3. Let $X$ be a nonempty compact subset of $E$. Assume that

(i) $K$ is continuous and close-valued on $\Lambda \times X$;

(ii) $C$ is u.s.c. on $X \times X$;

(iii) for any fixed $(\lambda, x) \in \Lambda \times X$, $f$ is lower $C(\lambda, x)$ continuous;

(iv) for each $\lambda \in \Lambda$ and $p \in P, S(\lambda, p) \neq \varnothing$.

Then, $\Pi$ is u.s.c. on $\Lambda \times P \times R^{+}$.

Proof. Let $\left(\lambda_{0}, p_{0}, \varepsilon_{0}\right) \in \Lambda \times P \times R^{+}$be any fixed point. Then, by (iv) and Lemma 3.2, $\Pi\left(\lambda_{0}, p_{0}, \varepsilon_{0}\right)$ is nonempty and closed. Furthermore, $\Pi\left(\lambda_{0}, p_{0}, \varepsilon_{0}\right)$ is nonempty and compact since $\Pi\left(\lambda_{0}, p_{0}, \varepsilon_{0}\right) \subseteq X$ and $X$ is compact. Let $\left(\lambda_{n}, p_{n}, \varepsilon_{n}\right) \rightarrow\left(\lambda_{0}, p_{0}, \varepsilon_{0}\right)$ and $x_{n} \in$ $\Pi\left(\lambda_{n}, p_{n}, \varepsilon_{n}\right)$. Observe that $\left\{x_{n}\right\} \subseteq X$ and $X$ is compact. We may assume that $x_{n} \rightarrow x_{0}$ for some $x_{0} \in X$. Then, by similar arguments as Lemma 3.2, we can show that $x_{0} \in$ $\Pi\left(\lambda_{0}, p_{0}, \varepsilon_{0}\right)$. This implies that $\Pi$ is u.s.c. at $\left(\lambda_{0}, p_{0}, \varepsilon_{0}\right)$. Then, by the arbitrary of $\left(\lambda_{0}\right.$, $\left.p_{0}, \varepsilon_{0}\right)$, we know that $\Pi$ is u.s.c. on $\Lambda \times P \times R^{+}$. This completes the proof.

Next, we give an example to illustrate Theorem 3.3.

Example 3.1. Let $E=R, X=[0,2], \Lambda=P=[0,1] \subseteq R, Y=R^{2}$. Let

$$
\begin{gathered}
e(x)=(1,0), \quad \forall x \in X, \\
C(\lambda, x)=\left\{(\xi, \eta) \in R^{2}: \eta \geq 0, \xi+\eta\left[\frac{2}{\pi} \arctan (\lambda x)\right] \geq 0\right\}, \quad \forall(\lambda, x) \in \Lambda \times X, \\
K(\lambda, x)=[\lambda x, x], \quad \forall(\lambda, x) \in \Lambda \times X, \\
f(p, x, y)=(p-x, x-y), \quad \forall(p, x, y) \in P \times X \times X .
\end{gathered}
$$

Then, it is easy to see that all the conditions of Theorem 3.3 are satisfied and so $\Pi$ is u.s.c. on $\Lambda \times P \times R^{+}$. Indeed, by simple computation, we have $\Pi(\lambda, p, \varepsilon)=[0, p+\varepsilon] \cap$ $[0,2]$ for all $(\lambda, p, \varepsilon) \in \Lambda \times P \times R^{+}$. Thus, $\Pi$ is u.s.c. on $\Lambda \times P \times R^{+}$.

\subsection{Metric characterization of well-posedness for (PSVQEP)}

In order to give metric characterizaton of well-posedness for (PSVQEP), we introduce the following notation.

Let $(\lambda, p) \in \Lambda \times P$ be given sequence. The approximating solution set of (PSVQEP) $\lambda$, $p$ is defined by, for each $\delta \geq 0$ and $\varepsilon \geq 0$,

$$
\begin{aligned}
\Omega_{\lambda, p}(\delta, \varepsilon) & =\bigcup_{\substack{\lambda^{\prime} \in B(\lambda, \delta) \cap \Lambda \\
p^{\prime} \in B(p, \delta) \cap P}} \Pi\left(\lambda^{\prime}, p^{\prime}, \varepsilon\right) \\
& \bigcup_{\substack{\lambda^{\prime} \in B(\lambda, \delta) \cap \Lambda \\
p^{\prime} \in B(p, \delta) \cap P}}\left\{x \in X: x \in K\left(\lambda^{\prime}, x\right) \text { and } f\left(p^{\prime}, x, y\right)+\varepsilon e(x) \in C\left(\lambda^{\prime}, x\right), \quad \forall y \in K\left(\lambda^{\prime}, x\right)\right\},
\end{aligned}
$$

where $B(a, r)$ denotes the closed ball centered at $a$ with radius $r$.

Clearly, we have, for every $(\lambda, p) \in \Lambda \times P$,

(i) $\Omega_{\lambda, p}(0,0)=\Pi(\lambda, p, 0)=S(\lambda, p)$;

(ii) $S(\lambda, p) \subseteq \Pi(\lambda, p, \varepsilon) \subseteq \Omega_{\lambda, p}(\delta, \varepsilon), \forall \delta, \varepsilon>0$;

(iii) if $0 \leq \delta_{1} \leq \delta_{2}$ and $0 \leq \varepsilon_{1} \leq \varepsilon_{2}$, then $\Omega_{\lambda, p}\left(\delta_{1}, \varepsilon_{1}\right) \subseteq \Omega_{\lambda, p}\left(\delta_{2}, \varepsilon_{2}\right)$.

Lemma 3.3. Assume that 
(i) for each $x \in X, K(\cdot, x)$ is continuous and close-valued;

(ii) for each $x \in X, C(\cdot, x)$ is u.s.c. and close-valued;

(iii) for each $x \in X, f(\cdot, x, \cdot)$ is continuous.

Then, for every $(\lambda, p) \in \Lambda \times P, S(\lambda, p)=\bigcap_{\delta>0, \varepsilon>0} \Omega_{\lambda, p}(\delta, \varepsilon)$.

Proof. Let $(\lambda, p) \in \Lambda \times P$ be any given sequence. Clearly, $S(\lambda, p) \subseteq \bigcap_{\delta>0, \varepsilon>0} \Omega_{\lambda, p}(\delta, \varepsilon)$. Thus, we only need to show that $\bigcap_{\delta>0, \varepsilon>0} \Omega_{\lambda, p}(\delta, \varepsilon) \subseteq S(\lambda, p)$. Indeed, if $x \in \bigcap_{\delta>0, \varepsilon>0} \Omega_{\lambda, p}(\delta, \varepsilon)$, then, for each $\delta>0$, and $\varepsilon>0, x \in \Omega_{\lambda, p}(\delta, \varepsilon)$. Thus, for each $n \in$ $N, x \in \Omega_{\lambda, p}\left(\frac{1}{n}, \frac{1}{n}\right)$ and, so there exists $\left(\lambda_{n}, p_{n}\right) \in \Lambda \times P$ with $\lambda_{n} \in B\left(\lambda, \frac{1}{n}\right)$ and $p_{n} \in B\left(p, \frac{1}{n}\right)$ such that

$$
x \in K\left(\lambda_{n}, x\right)
$$

and

$$
f\left(p_{n}, x, y\right)+\frac{1}{n} e(x) \in C\left(\lambda_{n}, x\right), \quad \forall y \in K\left(\lambda_{n}, x\right) .
$$

Clearly, $\lambda_{n} \rightarrow \lambda$ and $p_{n} \rightarrow p$ as $n \rightarrow \infty$. Since $K(\cdot, x)$ is u.s.c. and closed-valued, $K(\cdot, x)$ is closed and so $x \in K(\lambda, x)$. For each $y \in K(\lambda, x)$, since $K(\cdot, x)$ is l.s.c., there exists $y_{n} \in$ $K\left(\lambda_{n}, x\right)$ such that $y_{n} \rightarrow y$. Then, by (3.12), we have

$$
f\left(p_{n}, x, y_{n}\right)+\frac{1}{n} e(x) \in C\left(\lambda_{n}, x\right), \quad \forall n \in N .
$$

Since $C(\cdot, x)$ is u.s.c. and close-valued, $C(\cdot, x)$ is closed. Then, by the continuity of $f($, $x, \cdot)$, we get

$$
f(p, x, y) \in C(\lambda, x) .
$$

By the arbitrary of $y$, we know that $x \in S(\lambda, p)$ and this implies that $\bigcap_{\delta>0, \varepsilon>0} \Omega_{\lambda, p}(\delta, \varepsilon) \subseteq S(\lambda, p)$. Therefore, $S(\lambda, p)=\bigcap_{\delta>0, \varepsilon>0} \Omega_{\lambda, p}(\delta, \varepsilon)$. This completes the proof.

Lemma 3.4. Assume that

(i) $\Lambda$ and $P$ are finite dimensional;

(ii) $K$ is continuous and close-valued;

(iii) $C$ is u.s.c. and close-valued;

(iii) $f$ is continuous.

Then, for each $\delta>0$ and $\varepsilon>0, \Omega_{\lambda, p}(\delta$, $\varepsilon)$ is closed.

Proof. Let $\delta \geq 0$ and $\varepsilon \geq 0$ be given. Let $\left\{x_{n}\right\} \subseteq \Omega_{\lambda, p}(\delta, \varepsilon)$ be any sequence such that $x_{n} \rightarrow x$. Then, for each $n \in N$, there exist $\left(\lambda_{n}, p_{n}\right) \in \Lambda \times P$ with $\lambda_{n} \in B(\lambda, \delta)$ and $p_{n} \in$ $B(p, \delta)$ such that

$$
x_{n} \in K\left(\lambda_{n}, x_{n}\right)
$$


and

$$
f\left(p_{n}, x_{n}, y\right)+\varepsilon e\left(x_{n}\right) \in C\left(\lambda_{n}, x_{n}\right), \quad \forall y \in K\left(\lambda_{n}, x_{n}\right)
$$

Noting that $\Lambda$ and $P$ are finite dimensional, without loss of generality, we may assume that $\lambda_{n} \rightarrow \lambda_{0}$ and $p_{n} \rightarrow p_{0}$ for some $\lambda_{0} \in \Lambda$ and $p_{0} \in P$. It follows that

$$
d\left(\lambda_{0}, \lambda\right)=\lim _{n \rightarrow \infty} d\left(\lambda_{n}, \lambda\right) \leq \delta \text { and } d\left(p_{0}, p\right)=\lim _{n \rightarrow \infty} d\left(p_{n}, p\right) \leq \delta .
$$

Hence, $\lambda_{0} \in B(\lambda, \delta)$ and $p_{0} \in B(p, \delta)$. Since $K$ is u.s.c. and close-valued, $K$ is closed and so $x \in K(\lambda, x)$. For each $y \in K(\lambda, x)$, since $K$ is l.s.c., there exists $y_{n} \in K\left(\lambda_{n}, x_{n}\right)$ such that $y_{n} \rightarrow y$. Then, by (3.14), we have

$$
f\left(p_{n}, x_{n}, y_{n}\right)+\varepsilon e\left(x_{n}\right) \in C\left(\lambda_{n}, x_{n}\right), \quad \forall n \in N .
$$

Since $C$ is u.s.c. and close-valued, $C$ is closed. Then, by the continuity of $f$ and $e$, we get

$$
f\left(p_{0}, x, y\right)+\varepsilon e(x) \in C\left(\lambda_{0}, x\right)
$$

Thus, $x \in \Omega_{\lambda, p}(\delta, \varepsilon)$, and so $\Omega_{\lambda, p}(\delta, \varepsilon)$ is closed. This completes the proof.

The following theorem shows that the well-posedness in the generalized sense for (PSVQEP) can be characterized by considering the noncompactness of approximating solution set.

Theorem 3.4. Let $\Lambda$ and $P$ be finite dimensional. Assume that

(i) $K$ is continuous and close-valued;

(ii) $C$ is continuous and close-valued;

(iii) $f$ is continuous.

Then, (PQSVEP) is well-posed in the generalized sense, if and only if for every $(\lambda, p) \in$ $\Lambda \times P$

$$
\Omega_{\lambda, p}(\delta, \varepsilon) \neq \emptyset, \forall \delta, \varepsilon>0 \text {, and } \mu\left(\Omega_{\lambda, p}(\delta, \varepsilon)\right) \rightarrow 0 \text { as }(\delta, \varepsilon) \rightarrow(0,0) \text {. }
$$

Proof. Suppose that (PSVQEP) is well-posed in the generalized sense. Then, for each $(\lambda, p) \in \Lambda \times P, S(\lambda, p)$ is nonempty and compact. Clearly, $S(\lambda, p) \subseteq \Omega_{\lambda, p}(\delta, \varepsilon)$ for all $\delta$, $\varepsilon>0$. It follows that $\Omega_{\lambda, p}(\delta, \varepsilon) \neq \varnothing$ for all $\delta, \varepsilon>0$. Now we shall show that

$$
\mu\left(\Omega_{\lambda, p}(\delta, \varepsilon)\right) \rightarrow 0 \text { as }(\delta, \varepsilon) \rightarrow(0,0) .
$$

Observe that for each $\delta, \varepsilon>0$,

$$
H\left(\Omega_{\lambda, p}(\delta, \varepsilon), S(\lambda, p)\right)=\max \left\{h\left(\Omega_{\lambda, p}(\delta, \varepsilon), S(\lambda, p)\right), h\left(S(\lambda, p), \Omega_{\lambda, p}(\delta, \varepsilon)\right)\right\}=h\left(\Omega_{\lambda, p}(\delta, \varepsilon), S(\lambda, p)\right) .
$$

Taking into account the compactness of $S(\lambda, p)$, we get

$$
\mu\left(\Omega_{\lambda, p}(\delta, \varepsilon)\right) \leq 2 H\left(\Omega_{\lambda, p}(\delta, \varepsilon), S(\lambda, p)\right)+\mu(S(\lambda, p))=2 h\left(\Omega_{\lambda, p}(\delta, \varepsilon), S(\lambda, p)\right) .
$$

To prove (3.15), it is sufficient to show that

$$
h\left(\Omega_{\lambda, p}(\delta, \varepsilon), S(\lambda, p)\right) \rightarrow 0 \text { as }(\delta, \varepsilon) \rightarrow(0,0) .
$$


If $h\left(\Omega_{\lambda, p}(\delta, \varepsilon), S(\lambda, p)\right) \rightarrow 0$ as $(\delta, \varepsilon) \rightarrow(0,0)$, then there exist $l>0$ and $\delta_{n}>0, \varepsilon_{n}>0$ with $\delta_{n} \rightarrow 0, \varepsilon_{n} \rightarrow 0$, and $x_{n} \in \Omega_{\lambda, p}\left(\delta_{n}, \varepsilon_{n}\right)$ such that

$$
x_{n} \notin S(\lambda, p)+B(0, l), \quad \forall n \in N \text {. }
$$

As $x_{n} \in \Omega_{\lambda, p}\left(\delta_{n}, \varepsilon_{n}\right),\left\{x_{n}\right\}$ is an approximating sequence for (PSVQEP) $)_{\lambda, p}$. By the wellposedness in the generalized sense of (PSVQEP), there exists a subsequence $\left\{x_{n_{k}}\right\}$ of $\left\{x_{n}\right\}$ converging to some point of $S(\lambda, p)$. This contradicts (3.16) and so

$$
h\left(\Omega_{\lambda, p}(\delta, \varepsilon), S(\lambda, p)\right) \rightarrow 0 \text { as }(\delta, \varepsilon) \rightarrow(0,0) .
$$

Conversely, suppose that (3.15) holds. For each $(\lambda, p) \in \Lambda \times P$, by Lemma 3.4, $\Omega_{\lambda, p}$ $(\delta, \varepsilon)$ is closed for all $\delta, \varepsilon>0$. By Lemma 3.3, we have $S(\lambda, p)=\bigcap_{\delta>0, \varepsilon>0} \Omega_{\lambda, p}(\delta, \varepsilon)$. Since

$$
\mu\left(\Omega_{\lambda, p}(\delta, \varepsilon)\right) \rightarrow 0,
$$

the theorem on p.412 in [38] can be applied and one concludes that $S(\lambda, p)$ is nonempty, compact, and

$$
h\left(\Omega_{\lambda, p}(\delta, \varepsilon), S(\lambda, p)\right)=H\left(\Omega_{\lambda, p}(\delta, \varepsilon), S(\lambda, p)\right) \rightarrow 0 \text { as }(\delta, \varepsilon) \rightarrow(0,0) .
$$

Let $\left(\lambda_{n}, p_{n}\right) \rightarrow(\lambda, p) \in \Lambda \times P$ and $\left\{x_{n}\right\} \subseteq X$ be an approximating sequence for (PSVQEP $)_{\lambda, p}$ corresponding to $\left\{\left(\lambda_{n}, p_{n}\right)\right\}$. Then there exist $\varepsilon_{n}>0$ with $\varepsilon_{n} \rightarrow 0$ such that, for each $n \in N$,

$$
x_{n} \in K\left(\lambda_{n}, x_{n}\right)
$$

and

$$
f\left(p_{n}, x_{n}, y\right)+\varepsilon_{n} e\left(x_{n}\right) \in C\left(\lambda_{n}, x_{n}\right), \quad \forall y \in K\left(\lambda_{n}, x_{n}\right) .
$$

For each $n \in N$, let $\delta_{n}=\max \left\{d\left(\lambda_{n}, \lambda\right), d\left(p_{n}, p\right)\right\}$. Then $\delta_{n} \rightarrow 0$ and $x_{n} \in \Omega_{\lambda, p}\left(\delta_{n}, \varepsilon_{n}\right)$. It follows from (3.17) that

$$
d\left(x_{n}, S(\lambda, p)\right) \leq h\left(\Omega_{\lambda, p}\left(\delta_{n}, \varepsilon_{n}\right), S(\lambda, p)\right) \rightarrow 0 .
$$

Since $S(\lambda, p)$ is compact, there exists $\bar{x}_{n} \in S(\lambda, p)$ such that

$$
d\left(x_{n}, \bar{x}_{n}\right)=d\left(x_{n}, S(\lambda, p)\right) \rightarrow 0 .
$$

Again from the compactness of $S(\lambda, p),\left\{\bar{x}_{n}\right\}$ has a subsequence $\left\{\bar{x}_{n_{k}}\right\}$ converging to $\bar{x} \in S(\lambda, p)$. Hence, the corresponding subsequence $\left\{x_{n_{k}}\right\}$ of $\left\{x_{n}\right\}$ converges to $\bar{x}$. Therefore, (PSVQEP) is well-posed in the generalized sense. This completes the proof.

Example 3.2. Let $E, X, \Lambda, P, Y, e, K, C$, and $f$ be the same as in Example 3.1. Then, it is easy to see that the conditions (i)-(iii) of Theorem 3.4 are satisfied. Moreover, by simple computation, we have, for each $(\lambda, p) \in \Lambda \times P$ and each $\delta, \varepsilon \geq 0$ and each $\lambda^{\prime} \in$ $[\lambda-\delta, \lambda+\delta] \cap \Lambda$ and each $p^{\prime} \in[p-\delta, p+\delta] \cap P$,

$$
\Pi\left(\lambda^{\prime}, p^{\prime}, \varepsilon\right)=\left[0, p^{\prime}+\varepsilon\right] \cap[0,2] .
$$

It follows that

$$
\Omega_{\lambda, p}(\delta, \varepsilon)=\bigcup_{p^{\prime} \in[p-\delta, p+\delta] \cap P}\left(\left[0, p^{\prime}+\varepsilon\right] \cap[0,2]\right)=[0, p+\delta+\varepsilon] \cap[0,1+\varepsilon] \cap[0,2] .
$$


Thus, $\Omega_{\lambda, p}(\delta, \varepsilon) \neq \varnothing$ for all $\delta, \varepsilon>0$ and $\mu\left(\Omega_{\lambda, p}(\delta, \varepsilon)\right) \rightarrow 0$ as $(\delta, \varepsilon) \rightarrow(0,0)$. By Theorem 3.4, (PSVQEP) is well-posed in the generalized sense.

Indeed, by simple computation, we have $S(\lambda, p)=[0, p]$ for all $(\lambda, p) \in \Lambda \times P$. Suppose that $\left\{\left(\lambda_{n}, p_{n}\right)\right\} \subseteq \Lambda \times P$ with $\left(\lambda_{n}, p_{n}\right) \rightarrow(\lambda, p)$ and $\left\{x_{n}\right\}$ is an approximating sequence for (PSVQEP) $)_{\lambda, p}$ corresponding to $\left\{\left(\lambda_{n}, p_{n}\right)\right\}$. Then there exists $\left\{\varepsilon_{n}\right\} \subseteq R^{+}$with $\varepsilon_{n} \rightarrow 0$ such that $x_{n} \in \Pi\left(\lambda_{n}, p_{n}, \varepsilon_{n}\right)$, i.e., $x_{n} \in\left[0, p_{n}+\varepsilon_{n}\right] \cap[0,2]$. Since $p_{n} \rightarrow p \in P=$ $[0,1]$ and $\varepsilon_{n} \rightarrow 0$, there exists $n_{0} \in N$ such that, for each $n \geq n_{0}, p_{n}+\varepsilon_{n}<2$, and so $x_{n}$ $\in\left[0, p_{n}+\varepsilon_{n}\right]$. Since $\left\{x_{n}\right\}$ is bounded, it has a subsequence which converges to some point of $[0, p]=S(\lambda, p)$. Therefore, (PSVQEP) is well-posed in the generalized sense.

\section{Applications}

Since vector quasi-equilibrium problems contain vector quasi-variational inequality problems, vector quasi-optimization problems, and vector quasi-saddle point problems as special cases, we can derive from the result in Section 3, some consequences for such special cases. In this section, we discuss only some applications of our results to strong vector quasi-variational inequality problems and strong vector quasi-optimization problems.

Let $X$ and $Y$ be two real Banach spaces and $C$ be a closed convex cone in $Y$. Let $L(X$, $Y$ ) be the set of all the continuous linear operators from $X$ into $Y$. Let $e \in C$ be a fixed point.

\subsection{Strong vector quasi-variational inequality problems}

Let $F: X \rightarrow 2^{X}$ be a set-valued mapping and $G: X \rightarrow L(X, Y)$ a vector-valued mapping. We consider the following strong vector quasi-variational inequality problem (for short, SVQVIP): find $x \in X$ such that

$$
x \in F(x) \quad \text { and } \quad\langle G x, y-x\rangle \in C, \quad \forall y \in F(x) .
$$

Denote by $S$ the set of solutions for (SVQVIP). For each $\varepsilon \geq 0$, we denote by $\Pi(\varepsilon)$ the $\varepsilon$-solution set of (SVQVIP), i.e.,

$$
\Pi(\varepsilon)=\{x \in X: x \in F(x) \text { and }\langle G x, y-x\rangle+\varepsilon e \in C, \quad \forall y \in F(x)\} .
$$

Let $f(x, y)=\langle G x, y-x\rangle$. We can obtain the following results for (SVQVIP).

Theorem 4.1. (SVQVIP) is well-posed in the generalized sense, if and only if $S$ is a nonempty compact subset of $X$ and $\Pi$ is u.s.c. at 0.

Theorem 4.2. (SVQVIP) is well-posed, if and only if $S$ has a unique point and $\Pi$ is $u$. s.c. at 0 .

\subsection{Strong vector quasi-optimization problems}

Let $F: X \rightarrow 2^{X}$ be a set-valued mapping and $\phi: X \rightarrow Y$ be a vector-valued mapping. We consider the following strong vector quasi-optimization problem (for short, SVQOP):

$$
\min \varphi(x) \quad \text { subject to } x \in F(x) .
$$

A point $x_{0} \in X$ is called a strongly efficient solution of (SVQOP), if

$$
x_{0} \in F\left(x_{0}\right) \text { and } \varphi(y)-\varphi\left(x_{0}\right) \in C, \quad \forall y \in F\left(x_{0}\right) .
$$


Denote by $S$ the set of all strongly efficient solutions for (SVQOP). For each $\varepsilon \geq 0$, we denote by $\Pi(\varepsilon)$ the $\varepsilon$-strongly efficient solution set of (SVQOP) as follows:

$$
\Pi(\varepsilon)=\{x \in X: x \in F(x) \text { and } \varphi(y)-\varphi(x)+\varepsilon e \in C, \quad \forall y \in F(x)\} .
$$

Let $f(x, y)=\phi(y)-\phi(x)$. We can obtain the following results for (SVQOP).

Theorem 4.3. (SVQOP) is well-posed in the generalized sense, if and only if $S$ is a nonempty compact subset of $X$ and $\Pi$ is u.s.c. at 0.

Theorem 4.4. (SVQOP) is well-posed, if and only if $S$ has a unique point and $\Pi$ is $u$. s.c. at 0 .

\section{Acknowledgements}

This study was supported by the National Natural Science Foundation of China (11061023, 11071108), the Natural Science Foundation of Jiangxi Province (2010GZS0145, 2010GZS0151), the Youth Foundation of Jiangxi Educational Committee (GJJ10086) and the Scientific Research Fund Project of College of Science and Technology of Nanchang University (ZL-2010-01)

\section{Author details}

${ }^{1}$ College of Science and Technology, Nanchang University, Nanchang, Jiangxi 330029, People's Republic of China

2Department of Mathematics, Nanchang University, Nanchang, Jiangxi 330031, People's Republic of China

\section{Authors' contributions}

Q-YL carried out the study of well-posedness for parametric strong vector quasi-equilibrium problems, strong vector quasi-variational inequality problems and strong vector quasi-optimization problems, and participated in the sequence alignment and drafted the manuscript. S-HW conceived of the study, and participated in its design and coordination. All authors read and approved the final manuscript.

\section{Competing interests}

The authors declare that they have no competing interests.

Received: 13 May 2011 Accepted: 7 October 2011 Published: 7 October 2011

\section{References}

1. Blum, E, Oettli, W: From optimization and variational inequalities to equilibrium problems. Math Student. 63, 123-145 (1994)

2. Chen, GY, Huang, XX, Yang, XQ: Vector optimization: set-valued and variational analysis. Springer, Berlin, Heidelberg (2005)

3. Giannessi, F, (Ed): Vector variational inequalities and vector equilibria: mathematical theories. Kluwer Academic Publishers, Dordrecht (2000)

4. Tykhonov, AN: On the stability of the functional optimization problems. USSR J Comput Math Math Phys. 6(4), 28-33 (1966). doi:10.1016/0041-5553(66)90003-6

5. Levitin, ES, Polyak, BT: Convergence of minimizing sequences in conditional extremum problem. Soviet Math Dokl. 7, 764-767 (1966)

6. Bednarczuck, E, Penot, JP: Metrically well-set minimization problems. Appl Math Optim. 26, 273-285 (1992). doi:10.1007/ BF01371085

7. Crespi, GP, Guerraggio, A, Rocca, M: Well posedness in vector optimization problems and vector variational inequalities. J Optim Theory Appl. 132, 213-226 (2007). doi:10.1007/s10957-006-9144-2

8. Dontchev, AL, Zolezzi, T: Well-posed optimization problems, Lecture notes in mathematics. Springer, Berlin1543 (1993)

9. Huang, XX, Yang, XQ: Generalized Levitin-Polyak well-posedness in constrained optimization. SIAM J Optim. 17, 243-258 (2006). doi:10.1137/040614943

10. Miglierina, E, Molho, E, Rocca, M: Well-posedness and scalarization in vector optimization. J Optim Theory Appl. 126, 391-409 (2005). doi:10.1007/s10957-005-4723-1

11. Revalski, JP: Hadamard and strong well-posedness for convex programs. SIAM J Optim. 7, 519-526 (1997). doi:10.1137/ S1052623495286776

12. Zolezzi, T: Well-posedness criteria in optimization with application to the calculus of variations. Nonlinear Anal. 25, 437-453 (1995). doi:10.1016/0362-546X(94)00142-5

13. Zolezzi, T: Extended well-posedness of optimization problems. J Optim Theory Appl. 91, 257-266 (1996). doi:10.1007/ BF02192292

14. Ceng, LC, Hadjisavvas, N, Schaible, S, Yao, JC: Well-posedness for mixed quasivariational-like inequalities. J Optim Theory Appl. 139, 109-125 (2008). doi:10.1007/s10957-008-9428-9

15. Fang, YP, Hu, R: Parametric well-posedness for variational inequalities defined by bifunctions. Comput Math Appl. 53, 1306-1316 (2007). doi:10.1016/j.camwa.2006.09.009

16. Lignola, MB, Morgan, J: Approximating solutions and a-well-posedness for variational inequalities and Nash equilibria. Decision and Control in Management Science. pp. 367-378.Kluwer Academic Publishers, Dordrecht (2002)

17. Lignola, MB: Well-posedness and L-well-posedness for quasivariational inequalities. J Optim Theory Appl. 128(1), 119-138 (2006). doi:10.1007/s10957-005-7561-2 
18. Lucchetti, R, Patrone, F: A characterization of Tyhonov well-posedness for minimum problems, with applications to variational inequalities. Numer Funct Anal Optim. 3(4), 461-476 (1981). doi:10.1080/01630568108816100

19. Cavazzuti, E, Morgan, J: Well-posed saddle point problems. In: Hirriart-Urruty JB, Oettli W, Stoer J (eds.) Optimization, Theory and Algorithms. pp. 61-76. Marcel Dekker, New York (1983)

20. Lucchetti, R, Revalski, J, (Eds): Recent Developments in Well-Posed Variational Problems. Kluwer Academic Publishers, Dordrecht (1995)

21. Margiocco, M, Patrone, F, Pusillo, L: A new approach to Tykhonov well-posedness for Nash equilibria. Optimization. 40(4), 385-400 (1997). doi:10.1080/02331939708844321

22. Margiocco, M, Patrone, F, Pusillo, L: On the Tykhonov well-posedness of concave games and Cournot oligopoly games. J Optim Theory Appl. 112(2), 361-379 (2002). doi:10.1023/A:1013658023971

23. Morgan, J: Approximations and well-posedness in multicriteria games. Ann Oper Res. 137, 257-268 (2005). doi:10.1007/ s10479-005-2260-9

24. Lemaire, B: Well-posedness, conditioning, and regularization of minimization, inclusion, and fixed point problems. Pliska Stud Math Bulgar. 12, 71-84 (1998)

25. Lemaire, B, Ould Ahmed Salem, C, Revalski, JP: Well-posedness by perturbations of variational problems. J Optim Theory Appl. 115(2), 345-368 (2002). doi:10.1023/A:1020840322436

26. Yang, H, Yu, J: Unified approaches to well-posedness with some applications. J Global Optim. 31, 371-381 (2005). doi:10.1007/s10898-004-4275-1

27. Fang, YP, Hu, R, Huang, NJ: Well-posedness for equilibrium problems and for optimization problems with equilibrium constraints. Comput Math Appl. 55, 89-100 (2008). doi:10.1016/j.camwa.2007.03.019

28. Hu, R, Fang, YP: Levitin-Poylak well-posedness of variational inequalities. Nonlinear Anal. 72, 373-381 (2010). doi:10.1016/j.na.2009.06.071

29. Bianchia, M, Kassay, G, Pini, R: Well-posedness for vector equilibrium problems. Math Meth Oper Res. 70, 171-182 (2009). doi:10.1007/s00186-008-0239-4

30. Bianchia, M, Kassay, G, Pini, R: Well-posed equilibrium problems. Nonlinear Anal. 72, 460-468 (2010). do:10.1016/j. na.2009.06.081

31. Long, XJ, Huang, NJ, Teo, KL: Levitin-Polyak well-posedness for equilibrium problems with functional constraints. J Inequal Appl (2008). Article ID 657329, 14 pages

32. Kimura, K, Liou, YC, Wu, SY, Yao, JC: Well-posedness for parametric vector equilibtium problems with applications. J Indust Manag Optim. 4(2), 313-327 (2008)

33. Huang, NJ, Long, XJ, Zhao, CW: Well-posedness for vector quasi-equilibrium problems with applications. J Indust Manag Optim. 5(2), 341-349 (2009)

34. Li, MH, Li, SJ, Zhang, WY: Levitin-Polyak well-posedness of generalized vector quasi-equilibrium problems. J Indust Manag Optim. 5(4), 683-696 (2009)

35. Long, XJ, Huang, NJ: Metric characterizations of a-well-posedness for symmetric quasi-equilibrium problems. J Global Optim. 45, 459-471 (2009). doi:10.1007/s10898-008-9385-8

36. Gong, XH: Strong vector equilibrium problems. J Global Optim. 36, 339-349 (2006). doi:10.1007/s10898-006-9012-5

37. Aubin, JP, Ekeland, I: Applied nonlinear analysis. Wiley, New York (1984)

38. Kuratowski, K: Topology. Academic Press, New York1 and 2 (1968)

doi:10.1186/1687-1812-2011-62

Cite this article as: $L i$ and Wang: Well-posedness for parametric strong vector quasi-equilibrium problems with applications. Fixed Point Theory and Applications 2011 2011:62.

\section{Submit your manuscript to a SpringerOpen ${ }^{\circ}$ journal and benefit from:}

- Convenient online submission

- Rigorous peer review

- Immediate publication on acceptance

- Open access: articles freely available online

- High visibility within the field

- Retaining the copyright to your article

Submit your next manuscript at $\boldsymbol{s p r i n g e r o p e n . c o m ~}$ 